\title{
La interacción entre los derechos de propiedad intelectual y los procesos de innovación abierta aplicados en el mejoramiento vegetal moderno
}

\author{
$* * * *$ \\ Miguel A. Rapela \\ Maestría en Propiedad Intelectual, Facultad de Derecho, Universidad Austral. \\ Plataforma de Genómica y Mejoramiento UBATEC S.A./Facultad de Agronomía, \\ Universidad de Buenos Aires \\ mrapela@austral.edu.ar
}

Recibido: 29 de septiembre de 2020

Aceptado: 19 de octubre de 2020

\section{Resumen}

Los requerimientos de conocimientos y la necesidad de generación de nuevas invenciones tanto en productos como en procesos del mejoramiento vegetal moderno son altamente demandantes y en las fronteras de la ciencia. No hay en el sector público ni en el privado capacidad tecnológica autárquica capaz de dominar por completo emprendimientos de este tipo. En este marco general, los procesos de innovación abierta tanto pública/privada como privada/privada resultan clave para el éxito.

A pesar de las oportunidades que generan los procesos de innovación abierta aplicados al mejoramiento vegetal moderno, no se detecta un aumento en la tasa de innovación medida por el incremento promedio de la productividad de los cultivos. Una posible explicación de este hecho reside en que la base genética en la cual se está apoyando el mejoramiento vegetal moderno se ha estrechado de forma significativa por una falta de utilización o subutilización de los recursos genéticos. Los derechos de propiedad intelectual forman parte de las variables de esta compleja ecuación y su aplicación conduce a plantear tres hipótesis que fueron contrastadas con cuatro casos reales de innovación abierta aplicados en mejoramiento vegetal moderno en Argentina.

En ningún caso se observó que la supresión de los derechos de propiedad intelectual haya sido considerada una herramienta para fomentar la innovación (hipótesis 1). Por el contrario, lo que se observó es que la innovación se orientó hacia aquellos productos o procesos capaces de ser gestionados en forma innovadora por algún sistema de propiedad intelectual vigente, aunque la protección esté limitada por la velocidad del avance tecnológico (hipótesis 2). Una solución 
alternativa (hipótesis 3) es que el marco legal para fomentar el desarrollo deba reformularse de forma integral para adecuarlo a los desarrollos del mejoramiento vegetal moderno bajo procesos de innovación abierta. La decisión para una u otra alternativa es crítica, dado que de ella dependerá la tasa de innovación resultante de los cultivos.

Palabras clave: mejoramiento vegetal, innovación abierta, derechos de propiedad intelectual.

\title{
The Interaction Between Intellectual Property Rights and Open Innovation Processes Applied to Modern Plant Breeding
}

\begin{abstract}
The knowledge requirements and the need to generate new inventions in both products and processes of modern plant breeding are highly demanding and on the frontiers of science. There is no self-sufficient technological capacity in the public or private sector capable of completely dominating such undertakings. In this general framework, the processes of open innovation, both public/private, public/public and private/private, are key to success.

Despite the opportunities generated by open innovation processes applied to modern plant breeding, an increase in the innovation rate is not detected, as measured by the average increase in crop productivity. One possible explanation for this fact is that the genetic basis on which modern plant breeding is being supported has been significantly narrowed by a lack of utilization or underutilization of genetic resources.

Intellectual property rights are part of the variables of this complex equation and their application leads to three hypotheses that were tested against four real cases of open innovation applied in modern plant breeding in Argentina.

In no case it was observed that the suppression of intellectual property rights has been considered a tool to promote innovation (hypothesis \#1). On the contrary, what was observed is that the innovation was oriented towards those products or processes capable of being managed in an innovative way by some current intellectual property system, although protection is limited by the speed of technological advance. An alternative solution (hypothesis \#3), is that the legal framework to promote development should be reformulated in an integral way to adapt it to the developments of modern plant breeding under open innovation processes. The decision for one or the other alternative is critical since the innovation rate resulting from the crops will depend on it.
\end{abstract}

Key words: plant breeding, open innovation, intellectual property rights.

\section{A interação entre os direitos de propriedade intelectual e os processos de inovação aberta aplicados no melhoramento de plantas moderno}

\section{Resumo}

As exigências de conhecimento e a necessidade de gerar novas invençôes, tanto em produtos como em processos de melhoramento de plantas, são altamente exigentes e encontram-se nas 
fronteiras da ciência. Não há capacidade tecnológica autárquica no sector público ou privado que seja capaz de dominar completamente empreendimentos desse tipo. Neste quadro geral, os processos de inovação aberta, tanto público/privado como privado/privado, são a chave do sucesso. Apesar das oportunidades geradas pelos processos de inovaçáo aberta aplicados ao melhoramento moderno de plantas, não é detectado aumento na taxa de inovação medida pelo aumento médio da produtividade das culturas. Uma explicação possível para este facto é que a base genética sobre a qual o melhoramento vegetal moderno está a ser apoiado se reduziu significativamente devido à falta ou subutilização dos recursos genéticos.

Os direitos de propriedade intelectual são uma das variáveis desta complexa equação e a sua aplicaçáo leva a três hipóteses que foram testadas com quatro casos reais de inovação aberta aplicados no melhoramento de plantas modernas na Argentina.

Em nenhum caso se observou que a supressáo dos direitos de propriedade intelectual tem sido considerada um instrumento para promover a inovação (hipótese 1). Pelo contrário, o que foi observado é que a inovação foi orientada para aqueles produtos ou processos capazes de serem geridos de forma inovadora por algum sistema de propriedade intelectual existente, mesmo que a protecção seja limitada pela velocidade do progresso tecnológico (hipótese 2). Uma solução alternativa (hipótese 3), é que o quadro legal para promover o desenvolvimento deve ser reformulado de forma integral para o adaptar aos desenvolvimentos da moderna melhoramento de plantas em processos de inovação abertos. A decisão por uma ou outra alternativa é crítica, uma vez que a taxa de inovaçáo resultante das culturas dependerá disso.

Palavras chave: melhoramento vegetal, inovação aberta, direitos de propriedade intelectual.

\section{Introducción. Invención, innovación, innovación cerrada y abierta}

$\mathrm{Al}$ analizar la innovación abierta y la creación de valor en el mejoramiento vegetal, Müller y Campos (2020) sostienen que la evolución de la sociedad humana está estrechamente relacionada y depende del progreso que el hombre ha logrado en la agricultura, dado lo cual no es de extrañar que esta haya sido un terreno especialmente fértil para la innovación. En este marco, el objetivo del presente artículo es explorar la interacción existente entre los derechos de propiedad intelectual en variedades vegetales (en particular, derechos del obtentor y patentes de invenciones biotecnológicas) y los procesos de innovación abierta en el contexto general del mejoramiento vegetal moderno.

Esta triple interacción ha sido escasamente abordada en la literatura, debiéndose mencionar además de Müller y Campos (2020), los aportes de Aoki (2009), Brem, Nylund y Hitchen (2017), Byrum (2017), EuropaBio (2018), Henry (2019) y Oguamanam (2013). Una de las razones que pueden explicar el hecho de este relativo menor interés radica en que dicha interacción ha sido más analizada en el contexto de industrias económicamente relevantes, como, por ejemplo, la farmacéutica, la de hardware, la de software y no tanto en industrias 
estratégicamente sensibles, como la de semillas. Además, en los trabajos mencionados, la interacción ha sido analizada en forma descriptiva.

El presente trabajo intenta profundizar el análisis de las consecuencias y limitaciones de la interacción a los fines del mantenimiento de la tasa de innovación de los cultivos, confrontando modelos de gestión de la propiedad intelectual con casos reales.

A los fines de introducir el tema, debemos mencionar que el término "innovación” es ampliamente utilizado en la vida moderna, tanto para describir procesos o productos como en contextos de comunicación o de marketing empresario. Dado que la innovación está estrechamente ligada a la invención, a menudo sucede que ambos términos se usan indistintamente y son confundidos entre sí. ${ }^{1}$

Sin embargo, los términos “invención” e "innovación” no son sinónimos y se aplican a bienes y servicios diferentes. Sintetizando estas fuentes mencionadas, la "invención" es la creación de algo nuevo y es un término que hace referencia a la generación de una nueva idea o conocimiento, cuya finalidad es resolver un problema técnico específico, y a la creación, diseño o desarrollo de un producto, dispositivo, método que no ha existido antes. La invención también puede ser un nuevo proceso para producir un producto o puede ser una mejora sobre un producto o un producto existente.

La “innovación”, por su parte, es un término bastante más difícil de definir e interpretar. Siguiendo las definiciones de IICA (2019), OECD (2018) y Ruane (2019), la innovación es la comercialización de la invención y hace referencia a la introducción en el mercado de nuevos y valiosos productos, procesos, tecnologías, servicios, mejoras o rediseńo de los existentes. Las innovaciones pueden ser tecnológicas, sociales, organizacionales y/o institucionales y, en el presente artículo, únicamente nos ocuparemos de las primeras, las cuales, a su vez, comprenden innovaciones en productos y procesos. La innovación tecnológica, expresa Kalange (2020), puede, en síntesis, definirse como la transformación de una idea en una realidad.

Invención e innovación se diferencian por su forma de protección y regulación. Mientras que las invenciones pueden protegerse por las diversas herramientas de los derechos de propiedad intelectual (DPI) existentes, tales como los secretos co-

1 Ver, por ejemplo, Baregheh, Rowley y Sambrook (2009); Bledow et al. (2009); Boer y During (2001); Crossan y Apaydin (2010); Instituto Interamericano de Cooperación para la Agricultura [IICA] (2019); O'Sullivan y Dooley (2009); Organization for Economic Co-operation and Development [OECD] (2005; 2018); Rogers (2003); Ruane (2019); Schilling (2013); Tidd y Bessant (2009). 
merciales o industriales, modelos de utilidad, patentes y, para el caso de variedades vegetales, por los derechos del obtentor (DOV), las innovaciones usualmente se protegen mediante el registro y la autorización comercial para la venta.

Otro aspecto que las diferencia es que si bien las invenciones pueden tener su origen tanto en áreas académicas como en áreas empresariales, las innovaciones son casi exclusivamente pertenecientes a estas últimas. Estadísticamente, pocas invenciones se materializan en innovaciones. De hecho, entonces, puede haber invenciones que no serán innovaciones, pero es improbable que existan innovaciones tecnológicas que no hayan sido precedidas por una invención, sea tanto registrable como no registrable.

Los procesos de invención han estado más relacionados con el mundo académico que con el industrial y lo inverso ha ocurrido con los procesos de innovación. Sea en un caso o en el otro, los procesos de invención e innovación han sido tradicionalmente cerrados y basados en recursos propios, lo que hoy denominamos "innovación cerrada" (closed innovation). Estos procesos estuvieron en cierta medida relacionados con dos factores contrapuestos: a) falta de orientación empresarial de parte de la comunidad académica; b) falta de confianza en la capacidad inventiva de la academia de parte de la comunidad empresarial. En este sistema de gestión de la innovación, los proyectos se desarrollaban exclusivamente de principio a fin por medios de la propia organización, empresa o institución.

En el caso de las empresas, esto llevó a la creación de grandes áreas de investigación y desarrollo (IyD) con numerosos y calificados recursos humanos generadores de conocimientos propios. La innovación cerrada se caracterizó tanto por investigar, crear y desarrollar productos o servicios completamente dentro de la empresa como por el hecho de adoptar fuertes estrategias de protección basadas en el secreto industrial, la aplicación de diversos sistemas de DPI registrables y una relativa baja utilización de las herramientas de licenciamiento.

Con el objetivo de acelerar y agilizar los procesos de innovación, reducir el riesgo y eficientizar el uso de recursos humanos y económicos, los procesos de innovación cerrada están siendo acompañados o aun reemplazados por procesos de innovación abierta (open innovation). Este moderno concepto y paradigma fue introducido por Henry Chesbrough y se basa en el supuesto de que para avanzar en innovación tecnológica, llegar más rápido y a menor costo al mercado, las empresas pueden y deben utilizar ideas y caminos tanto internos como externos. ${ }^{2}$ En la innovación abierta, los proyectos pueden originarse dentro o fuera de la

2 Entre muchos autores, ver la extensa obra sobre innovación abierta de Chesbrough (2003; 2006; 2011; 2019); Chesbrough y Bogers (2014); Chesbrough, Vanhaverbeke y West (2006). 
empresa, incorporarse al principio o en fases intermedias del proceso de innovación y ser lanzados al mercado a través de la misma compañía o a través de otras empresas, mediante la utilización de patentes o transferencias de tecnología vía licenciamiento. Asimismo, la IyD interna de la empresa también existe, pero únicamente se centra en la creación de conocimiento que no existe o no se puede acceder fuera de la empresa. Claramente, y para el caso de la vinculación público/ privada, el concepto de "innovación abierta" generó un virtuosismo bidireccional más avanzado que el concepto de "innovación cerrada", ya que las invenciones del sector académico también pueden disponer de un terreno más fértil para convertirse en innovaciones. Además, así como las invenciones constituyen el basamento de las innovaciones, el desarrollo es el puente nexo que une a las dos. De esta manera, un concepto de gestión del conocimiento de reciente aparición y enteramente aplicable a la innovación abierta une a la invención (investigación) con el desarrollo y la innovación en una fórmula integral $\mathrm{I}+\mathrm{D}+\mathrm{I}$ como superadora del anterior concepto de $\mathrm{I}+\mathrm{D}$.

Probablemente, por una confusión de términos tales como "código abierto" u open access de la industria de software, a veces se ha considerado a los DPI como antagónicos al concepto de "innovación abierta”. Objetivamente esto no es así, por el contrario, en lo concerniente a los DPI, la innovación abierta no difiere sustancialmente de la innovación cerrada. "Innovación abierta no es sinónimo de código abierto”, expresan Müller y Campos (2020, p. 85). En la innovación abierta, todos los tipos de DPI pueden tener cabida y ser aplicados de manera tan fuerte como en los procesos de innovación cerrada. Lo que difiere entre la innovación cerrada y la abierta es la forma de gestionar los DPI, la cual hace un uso extensivo de las licencias como base para compartir tecnologías o transferirlas (EuropaBio, 2018), y puede contener, en algunos casos, áreas de libre disponibilidad de los bienes intangibles generados (Hall, 2010). Asimismo, bajo el concepto de "innovación abierta", las empresas no solamente son generadoras de DPI, sino también usuarios activos de DPI (Pénin y Neicu, 2018).

El mejoramiento vegetal tradicional desarrollado en empresas ha estado históricamente relacionado con la academia, pero en un contexto de innovación cerrada. ${ }^{3}$ El mejoramiento vegetal moderno (ver su desarrollo en el siguiente apartado), sin embargo, es un campo interdisciplinario en el cual se conjuga la alta tecnología de vanguardia a los fines de la mejor utilización y aprovechamiento de los recursos genéticos existentes. Es por esta razón que la interrelación entre esta tecnología y la propiedad intelectual en procesos de innovación abierta

3 Ver, por ejemplo, el análisis de Müller y Campos (2020). 
constituye todo un desafío, lo cual es el punto central que se explorará en el presente artículo.

No es objeto de este artículo el estudio o profundización de los diferentes tipos de DPI aplicables en vegetales ni examinar sus aspectos regulatorios. A tales fines, se remite a textos clásicos en la materia, como Botana Agra (2017a; 2017b); Correa (2014; 2015); Fernando Magarso y Lema Devesa (2002); Lema Devesa y Fernando Magarzo (2002); Sánchez Herrero (2007); Rapela (2000); Rapela y Schötz (2006).

\section{Coevolución del mejoramiento vegetal y el concepto de "innovación": tres hipótesis}

Por medio de la domesticación de las especies cultivadas basada exclusivamente en la selección visual de los mejores ejemplares de plantas, el mejoramiento vegetal fue practicado en forma empírica por los seres humanos desde el momento mismo de la transición del nomadismo (paleolítico) al sedentarismo (neolítico). En 1866, y tras el trabajo de Gregor Mendel -que recién sería reconocido a principios del siglo XX-, comienza la era científica del mejoramiento vegetal, aunque también basada en la selección por el fenotipo, y, más de cien años después -en 1970-, se comenzó a transitar la era fenogenotípica del mejoramiento vegetal, la cual caracterizó a la agricultura 3.0. A una velocidad vertiginosa, el siglo XXI se abre a una era genómica del mejoramiento vegetal, en la cual la estructura fina del material hereditario es completamente accesible, permitiendo la recombinación dirigida mediante la selección de genes específicos utilizando herramientas moleculares para rastrear la variación dentro del genoma. Esto es lo que se denomina "mejoramiento vegetal moderno", el cual caracteriza a una agricultura 4.0, cuyo objetivo es obtener nuevas variedades de plantas basado en la selección genómica y fenómica generada en big data. La enorme cantidad de puntos de información que puede llegar a trillones de datos para la selección de una sola variedad vegetal hace necesario usar inteligencia artificial a los fines de conjugar una visión y análisis completo del problema a través de una interacción ser humano-computadora nunca abordada. Este conocimiento "fino" del genoma y el incipiente entendimiento profundo de la relación entre genotipo y fenotipo están permitiendo la aplicación de la edición génica a los fines de poder modificar la expresión de los genes clave relacionados con la productividad y la tolerancia a distintos tipos de estrés bióticos y abióticos (Rapela, 2020).

El avance genético y biotecnológico de la "agricultura 3.0" estuvo fuertemente marcado por un concepto de "innovación cerrada" fundamentalmente liderado 
por compañías privadas, las cuales, mediante la incorporación de significativos recursos humanos y económicos, llegaron a la concreción práctica de la transgénesis que modificó la agricultura de muchos países, en particular en todo el continente americano. En un modelo muy diferente, la era genómica, junto con las técnicas de selección asistida por marcadores moleculares y la edición génica que forman el corazón de la "agricultura 4.0", está liderada fundamentalmente por centros académicos públicos y ahora son los propios investigadores los que marcan la ruta de la futura comercialización de los nuevos productos mediante conceptos estrechamente basados en la innovación abierta (Rapela, 2019a).

La "agricultura 3.0" parece haber pasado a toda velocidad evolucionando hacia una "agricultura 4.0" con elementos centrales diferentes: a) de conocimientos monopólicos a democratización de los conocimientos; b) de privada a pública; c) de empresas a universidades; d) de acceso físico al recurso a acceso a la información; e) de licencias exclusivas a licencias no exclusivas (Rapela, 2019a).

En lo referente a los DPI, el mejoramiento vegetal de la agricultura 3.0 se caracterizó porque los productos directos del mejoramiento vegetal $-\mathrm{o}$ sea, las variedades de plantas nuevas, distintas, uniformes y estables- podían ser protegidas por un título de propiedad intelectual sui generis conocido como DOV. Las invenciones biotecnológicas, por su parte, podían calificar para ser protegidas mediante patentes una vez cumplidos los requisitos de novedad absoluta, altura inventiva y aplicación industrial. Ambos derechos pueden coexistir y, de hecho, hay innumerables variedades protegidas mediante un DOV que contienen una o más construcciones genéticas patentadas obtenidas en laboratorio. El problema es que estos paradigmas están fuertemente desafiados en el mejoramiento vegetal moderno de la agricultura 4.0, a tal punto que ya existen desarrollos genéticos concretos que no pueden ser protegidos por ningún sistema de DPI en curso (Rapela, 2019b).

No existen dudas, expresan Müller y Campos (2020), que para cumplir con el enorme desafío de proporcionar alimentos nutritivos y asequibles a la humanidad y, al mismo tiempo, reducir la huella ambiental de la agricultura se necesitan nuevos modelos de innovación. En este contexto, las empresas semilleras están adoptando la innovación abierta para aprovechar recursos humanos disponibles fuera de ellas y, asimismo, incrementar su capacidad para generar, adoptar, desarrollar y llevar al mercado tecnologías novedosas.

El mejoramiento genético de los cultivos ha sido imprescindible para garantizar la seguridad alimentaria de una población mundial en permanente crecimiento. De hecho, el mejoramiento vegetal es una de las claves fundamentales para interpretar la razón por la cual las predicciones de Robert Malthus no resul- 
taron, hasta el momento, acertadas. De no haber existido el mejoramiento vegetal realizado durante el siglo XX y lo que va del XXI, difícilmente la provisión de alimentos del mundo podría sustentar a una población superior a los 2000 millones de personas (Henry, 2019; Müller y Campos, 2020; Rapela, 2020).

Pero si bien no existen dudas respecto a que la innovación es un motor clave del crecimiento económico, sí las hay respecto a si los DPI -como las patentes, modelos de utilidad, derechos de autor o DOV- constituyen una herramienta eficaz para incentivar la innovación al permitir que las empresas y las instituciones académicas capturen una proporción de los beneficios de sus inversiones en investigación y estos sirvan para realimentar el desarrollo de mejores invenciones.

Como correctamente expresa Eidi Williams (2010), en muchas áreas del conocimiento mensurar este dilema es sumamente complejo, dado que a menudo los cambios tecnológicos son acumulativos, es decir, que el desarrollo del producto es el resultado de varios pasos de invención e investigación. En los mercados donde la invención es acumulativa, expresa esta autora, la efectividad general de los DPI en la promoción de la innovación también depende de una segunda pregunta menos estudiada: ¿los DPI sobre las tecnologías existentes obstaculizan o favorecen la innovación posterior?

Uno de los autores más conocidos que defienden lo primero es Matt Ridley ${ }^{4}$ (2010; 2020), quien sostiene la hipótesis de que la innovación ocurre por prueba y error, que la práctica lidera a los avances científicos y que los DPI no constituyen una herramienta para favorecerla (hipótesis 1). Aplicado al tema que trata aquí, Ridley $(2010$; 2020) argumenta que la experiencia empírica de prueba y error del mejoramiento vegetal pre-Mendel, la cual precedió a cualquier teoría válida sobre genética, constituyó el cimiento de la innovación, y que esta última no se sustentó en la base teórica científica pos-Mendel. En forma general, Ridley (2010; 2020) rechaza a los DPI como herramienta para la innovación, ya que muestran repetidamente que han retrasado el florecimiento humano más de lo que lo han ayudado y que los monopolios protegidos por el Gobierno para algunos inventos retrasan la innovación, desperdiciando recursos preciosos en peleas por patentes en lugar de luchas competitivas para inventar la próxima gran cosa. La mayoría de las veces solo reflejan quién fue lo suficientemente astuto o bien conectado con la oficina de patentes. La conclusión, expresa Ridley, es que los DPI son barreras para la búsqueda de la innovación y un lastre para la economía, y no una bendición necesaria para la innovación que los economistas modernos y los estudiosos

4 Periodista, economista y hombre de negocios de Inglaterra, miembro de la Cámara de los Lores. Es autor de numerosos libros en los que sustenta sus hipótesis en cuanto a innovación. 
del derecho quieren hacer creer. Según Ridley (2010; 2020), no existen indicios de una "falla del mercado" en la innovación a la espera de ser rectificada por la propiedad intelectual, mientras que existe una amplia evidencia de que las patentes y los derechos de autor obstaculizan la innovación (Book, 2020). ${ }^{5}$

Resulta válido suponer que si la hipótesis de Ridley $(2010$; 2020) respecto al efecto negativo de los DPI sobre la innovación fuera acertada, la tasa de innovación en mejoramiento vegetal medida por el incremento promedio de la productividad de los cultivos debería estar estancada o aun en disminución. De hecho, justamente eso es lo que está ocurriendo. En un exhaustivo análisis de fuentes internacionales públicas y privadas incuestionables, Rapela (2019a) señala que, en la última década, han empezado a hacerse claras señales de alarma sobre los crecimientos productivos de los diferentes cultivos. Numerosos estudios muestran que alimentar y dar energía al mundo requerirá que la producción agrícola entre los ańos 2010 y 2050 se incremente al doble, y para llegar a esta cifra se necesita una tasa de crecimiento de la productividad de los principales cultivos del orden del $2,4 \%$ por ańo. Pero las investigaciones privadas indican que las mejoras promedio del rendimiento de maíz, arroz, trigo y soja están con un crecimiento del 1,2\%, o sea, justo la mitad de lo necesario. Los informes de la Organización para la Cooperación y el Desarrollo Económicos (OECD, por sus siglas en inglés) son coincidentes y señalan que el crecimiento promedio anual de la agricultura en el período 2003-2012 fue del 2,1\%, el cual disminuyó a 1,5\% para el período 2013-2022, datos que fueron ratificados por esta organización junto con la Organización de las Naciones Unidas para la Alimentación y la Agricultura (FAO, por sus siglas en inglés) en el último informe para las proyecciones 2019-2028 (OECD-FAO, 2019). Otros reportes recientes, como los realizados por el Virginia Tech's College of Agriculture and Life Sciences, que fueron presentados durante el World Food Prize en Des Moines, Iowa (Estados Unidos), difieren en los valores absolutos, pero no en la diferencia. De acuerdo con esta institución, es necesario un incremento anual de la productividad de los cultivos del $1,73 \%$ para cubrir toda la demanda de alimentos, fibra y bioenergía hasta el año 2050, pero estos niveles solo se cumplen en China y en el sureste de Asia, son inferiores en Estados Unidos, Europa y Latinoamérica y no llegan al 1\% en los países menos desarrollados (Virginia Tech, 2019).

5 En esta cita, Bock (2020) hace un análisis de las propuestas de Matt Ridley desestimando la relación entre derechos de propiedad intelectual e innovación, seńalando, entre otras cosas, el contradictorio hecho de que Ridley ha vendido millones de ejemplares de sus libros, los cuales están protegidos por derechos de autor por los cuales recibe regalías. 
En contraposición a la hipótesis de Ridley $(2010 ; 2020)$ de que los DPI obstaculizan la innovación, Rapela (2019a) conjetura que el complejo entramado de tratados, convenciones, protocolos, acuerdos internacionales y normativas regionales y nacionales que se aplican sobre las variedades vegetales y no los DPI en sí mismos son la causa del estancamiento señalado en la tasa de innovación (hipótesis 2).

Para este autor, la innovación, el desarrollo científico y técnico de variedades vegetales modernas, incluyendo microrganismos beneficiosos, el acceso y uso de los recursos fitogenéticos y el desarrollo de invenciones biotecnológicas y la bioseguridad están regulados a nivel mundial, regional y nacional por una enorme cantidad de legislaciones internacionales, regionales y nacionales. La interpretación en forma conjunta y combinada de estas es por demás dificultosa debido a los solapamientos, vacíos, ambigüedades, contradicciones y falta de acoplamiento mutuo. Basta señalar que mientras que el sistema de DOV es open source, todos los tratados de recursos fitogenéticos que regulan el acceso al material esencial del mejoramiento vegetal son close source. El cuadro es aún más complejo ya que una serie de desarrollos científicos que se aplican en mejoramiento vegetal en forma amplia -en particular las técnicas de edición génica- han provocado que varios de esos marcos regulatorios internacionales hayan quedado obsoletos. La hipótesis que plantea Rapela (2019a) es que estos hechos están relacionados; el entramado de legislaciones y regulaciones que se aplican sobre el desarrollo de las variedades vegetales modernas y microrganismos beneficiosos es la causa que está afectando el acceso y uso de los recursos genéticos y a la investigación y desarrollo en genética y mejoramiento vegetal, y el efecto provocado es el estancamiento del crecimiento de la productividad, es decir, la tasa de innovación. En forma similar, Dederer (2020) ha planteado que en vista de todas estas complejidades e incertidumbres, los legisladores deben monitorear continuamente la situación fáctica y legal y estar preparados para posibles enmiendas a las actuales legislaciones de patentes y DOV.

Dicho de otra manera, mientras que para Ridley $(2010 ; 2020)$ el estancamiento y la disminución de la tasa de innovación se debe a los DPI, para Rapela (2019a) esto se debe a la existencia de un marco altamente complejo, desarticulado e independiente de legislaciones sobre DPI, recursos genéticos y asuntos regulatorios que obstaculizan la innovación y ejercen una acción conjunta negativa sobre un único objeto al cual se aplican (la variedad vegetal). De tal forma, ambas hipótesis difieren en la posible salida. Mientras que para Ridley $(2010 ; 2020)$ la solución a la crisis del incremento productivo de los cultivos estaría en la eliminación de los DPI, para Rapela la solución se focaliza en desarrollar un nuevo y 
único marco integral de DPI para todo el germoplasma vegetal que permita un fomento concreto a la innovación, lo cual es algo completamente diferente.

Finalmente, entre ambas hipótesis se encuentra la propuesta por Lizaso y Schötz (2017): la solución más pragmática se basa en el uso de los instrumentos de DPI ya disponibles, pero bajo modernos sistemas de gestión (hipótesis 3). En un profundo y extenso análisis de plataformas abiertas en modernos procesos de innovación, Lizaso y Schötz (2017) sostienen que estos últimos evolucionaron aplicando mecanismos y herramientas híbridas de cocreación, incluyendo plataformas, aplicativos y nuevos tipos de estructuras organizacionales intermedias conocidas, tales como open innovation accelerators (OIA) y patent aggregations organizations (PAO). En particular, proponen que esta segunda estructura es adecuada al contemplar tanto: a) los propósitos que se pretenden alcanzar a través del objetivo general del proyecto; b) las exigencias de implementación en términos de factores críticos de éxito; y c) los criterios de implementación. Aplicado al análisis puntual del mejoramiento vegetal y desde el punto de vista de los DPI, estos autores sostienen que la innovación abierta sobre nuevas variedades vegetales que, a su vez, pueden contener invenciones biotecnológicas patentadas presenta un "horizonte promisorio" para el desarrollo tanto de OIA como de PAO. Estas posibilidades están reafirmadas por expertos de importantes compañías semilleras (Byrum, 2017).

También se puede agregar, como expresa Hall (2010), que en estas modernas plataformas los DPI se pueden utilizar a la defensiva para negociar licencias cruzadas con otras empresas en la industria o instituciones académicas que posean tecnologías complementarias, evitando así litigios mutuos, como se aplica por un gran número de empresas en las industrias de semiconductores, hardware y software informático. Asimismo, expresa este autor, los DPI pueden permitir la liberación selectiva al público de patentes que no están siendo explotadas, ${ }^{6} \mathrm{o}$ financiar activamente la investigación académica, sin especificar con precisión el objetivo o el área de la investigación. ${ }^{7}$

\section{Mejoramiento vegetal moderno e innovación abierta: análisis de casos}

A continuación se analizarán cuatro casos reales de Argentina, contrastando las tres hipótesis de trabajo para fomentar la innovación abierta en mejoramiento ve-

6 Caso Intel (Hall, 2010).

7 Caso Millennium, que ha otorgado licencias de genes a empresas farmacéuticas para aplicaciones específicas, al tiempo que conserva los derechos de todas las demás aplicaciones (Hall, 2010). 
getal moderno: a) eliminar los DPI (Ridley, 2010; 2020); b) gestionar de forma novedosa los DPI haciendo uso del marco normativo vigente (Lizaso y Schötz, 2017); c) desarrollar un nuevo sistema integrado sui generis de protección para todo tipo de germoplasma vegetal (Rapela, 2019a).

La selección de los casos ha sido al azar, pero condicionada por dos aspectos: 1) debían ser casos en los que las instituciones participantes fueran de amplio conocimiento y reconocimiento; 2) debían ser casos en los cuales fuese posible contar con información clave y comprobable "desde adentro" y factible de ser publicada con la autorización respectiva. De ninguna manera se está intentando decir que estos cuatro casos agotan los ejemplos a nivel nacional en la materia.

\section{Caso 1. Plataforma de Genómica y Mejoramiento (PGM) ${ }^{8}$}

La PGM está ubicada en la Cátedra de Bioquímica de la Facultad de Agronomía de la Universidad de Buenos Aires (UBA) y cuenta con la gestión y vinculación tecnológica de UBATEC S.A. La PGM es la plataforma de análisis genómico más avanzada de Argentina y su objetivo es asistir, complementar y ayudar a la industria nacional de mejoramiento vegetal y animal para el incremento de su competitividad, proporcionando un servicio de alto nivel de recursos humanos y tecnológicos. El proyecto es fruto de una política del Estado nacional y cuenta con financiamiento mediante la herramienta de Fortalecimiento a la Innovación Tecnológica de Aglomerados Productivos (FIT-AP) otorgado a través de la Agencia Nacional de Promoción Científica y Técnica.

Al día de hoy, once empresas de Argentina y una de Paraguay están recibiendo apoyo de la PGM, particularmente en las áreas de descubrimiento de genes, caracterización de germoplasma, mejoramiento asistido por marcadores y registro de propiedad para acceder a un DOV, lo cual es obligatorio para el cultivo de soja. En el momento de la firma de cada convenio, UBATEC S.A. y la empresa suscriben un estricto acuerdo de confidencialidad que no permite ni el uso ni la difusión de ningún resultado del servicio sin consentimiento previo de las partes. Cada proyecto es una unidad independiente con su presupuesto y plazo de ejecución. En casi la totalidad de los casos, además, la empresa proporciona las muestras de análisis bajo codificación.

Las empresas se benefician con un moderno, ágil y no burocrático concepto de innovación abierta púbico/privada gestionado por una sociedad anónima cuya dirección es tripartida ente la UBA, el Gobierno de la Ciudad de Buenos Aires y las cámaras empresariales, que les permite ahorrar enormes sumas de re-

8 El autor del presente trabajo es el actual director de Vinculación Tecnológica de la PGM. 
cursos económicos para sus planes de mejoramiento al no tener que disponer de instalaciones, equipamiento y recursos humanos para investigación y desarrollo de vanguardia. Hasta el momento, la política de la PGM es la de no involucrarse en la propiedad intelectual de las variedades vegetales o invenciones biotecnológicas que se puedan desarrollar, pero colabora en el asesoramiento y su gestión a solicitud de la empresa y a favor exclusivo de esta.

\section{Caso 2. Bioheuris S.A. ${ }^{9}$}

Es una start up biotecnológica de capital nacional ubicada en la ciudad de Rosario, la cual combina herramientas de biología sintética y edición génica para desarrollar cultivos resistentes a herbicidas. La empresa utiliza diseńo racional y evolución dirigida para primero identificar mutaciones que confieren resistencia a herbicidas y luego introducirlas en los cultivos mediante técnicas de edición génica.

Bioheuris ha firmado acuerdos de tipo privado/privado con pymes semilleras nacionales, quienes se benefician de un ambiente de innovación abierta win-win por el cual pueden disponer de acceso a tecnología de punta y, por su parte, Bioheuris puede aplicar esas tecnologías de punta sobre germoplasma elite de nivel competitivo comercial y con ello ahorrar años de desarrollo en ambos sentidos. En edición génica, Bioheuris trabaja mediante licencias exclusivamente para $\mathrm{I}+\mathrm{D}$ con Benson Hill Biosystems, una de las compañías más importantes de edición génica a nivel mundial, la cual es una de las únicas que posee tecnologías propietarias en este campo. También están trabajando con otros proveedores de nucleasas.

Los acuerdos Bioheuris/empresas semilleras serán contractuales e incluirán cláusulas de confidencialidad. La gestión de los DPI será, en principio, con el modelo de coexistencia; Bioheuris gestionará la protección de aquellas invenciones con posibilidades de patentamiento, mientras que las empresas tramitarán la protección de las variedades editadas mediante DOV. La licencia comprenderá ambos derechos registrales. En los casos de aquellos países - en particular Argentina- donde las oportunidades de patentamiento sean extremadamente bajas, la comercialización de las variedades incluirá el reconocimiento no registral de la invención a favor de Bioheuris.

Bioheuris, además, selecciona a sus países blancos con dos premisas: a) deben tener una política regulatoria clara respecto a la comercialización de las variedades vegetales editadas, como es el caso de Argentina, Brasil, Colombia y Estados

9 Síntesis elaborada a partir de la información proporcionada por el Dr. Carlos Pérez, socio fundador y director de Estrategia de Bioheuris S.A. 
Unidos; y b) deben estar adheridos al Tratado de Cooperación de Patentes. En este último caso, la excepción lo constituye justamente Argentina.

Bioheuris no hace uso de recursos genéticos (RRGG) en sus proyectos.

\section{Caso 3. Bioceres ${ }^{10}$}

Bioceres S.A. se encuentra ubicada en el Centro Científico Tecnológico de Rosario, el cual alberga, además, a catorce institutos de investigación del Consejo Nacional de Investigaciones Científicas y Técnicas (CONICET). La empresa de Investigación y Desarrollo $(\mathrm{I}+\mathrm{D})$ de Bioceres es INDEAR.

En un ambiente de innovación abierta, el socio estratégico de Bioceres es el CONICET, siendo el objeto principal de esta colaboración el desarrollo de múltiples eventos biotecnológicos y su aplicación en la protección de cultivos, principalmente soja y trigo. Mediante esta asociación público/privada, la dupla CONICET/Bioceres ha complementado sus capacidades para proveer servicios de $\mathrm{I}+\mathrm{D}+\mathrm{I}$ y facilitar el desarrollo de nuevas tecnologías y productos de Bioceres y de empresas fuera del grupo, proporcionando capacidades biotecnológicas de vanguardia y know-how especializado requeridos para el desarrollo de productos a costos competitivos.

La principal capacidad de Bioceres consiste en acelerar, de forma eficiente en términos de capital, el lanzamiento al mercado de las tecnologías prometedoras desarrolladas por grupos o instituciones científicas reconocidas internacionalmente. Además de la colaboración con el CONICET, se destacan las alianzas de Bioceres con la Universidad Nacional del Litoral, el Instituto Leloir, el Instituto Nacional de Tecnología Agropecuaria (INTA), la Universidad de Illinois y la Universidad de Australia.

En 2003, Bioceres celebró un acuerdo de investigación y desarrollo de la tecnología $\mathrm{HB}^{\oplus}{ }^{\oplus}$ con el CONICET y la Universidad Nacional del Litoral. Esta tecnología se refiere a un gen de girasol que confiere tolerancia a la sequía en cultivos y ayuda a aumentar el rendimiento en un promedio del 13\% al 19\% para múltiples cultivos en diferentes estaciones y condiciones de crecimiento, incluidos los episodios de sequía esporádica. $\mathrm{HB}^{\circledR}$ también puede proporcionar un mayor rendimiento sin afectar negativamente los rendimientos en condiciones óptimas de crecimiento, que es un factor distintivo e importante en comparación con otras tecnologías de tolerancia al estrés.

La estrategia de gestión de los DPI seleccionada por Bioceres fue por vía del

10 Síntesis elaborada a partir de la información proporcionada por la Dra. Gloria Montarón Estrada, directora de Asuntos Legales y Propiedad Intelectual de Bioceres S.A. 
patentamiento de las invenciones biotecnológicas. La tecnología $\mathrm{HB}^{\circledR}$ está protegida vía patentes en al menos once países, entre ellos, los principales países del espacio agro-business hasta el año 2033.

Bioceres no hace uso de RRGG en sus proyectos.

\section{Caso 4. Estación Experimental Agroindustrial Obispo Colombres (EEAOC) ${ }^{11}$} La EEAOC es un organismo autárquico del Gobierno de la provincia de Tucumán, que se sostiene con el aporte de los sectores agrícolas y agroindustriales de la provincia a través de una tasa de servicio obligatoria fijada por ley que se les cobra a aquellos sectores para los cuales trabaja. De hecho, es un organismo estatal gestionado por los propios productores, financiable con recursos de la producción y nutrido del conocimiento más avanzado disponible en el mundo.

Este organismo tiene programas de mejoramiento genético en caña de azúcar, soja, poroto, garbanzo, trigo y cítricos que se desarrollan mediante convenios de vinculación tecnológica y/o convenios de comercialización con varias empresas. Estos convenios, en muchos casos, implican una contraparte económica por parte de las empresas para ayudar al desarrollo de las variedades.

Dado que en variedades vegetales no hay actividades relacionadas con invenciones biotecnológicas, el único DPI que gestiona la empresa es el DOV. Según los casos, la EEAOC puede realizar el registro oficial o, a veces, es llevado a cabo por la empresa que formó parte del acuerdo. En todos los casos, la EEAOC participa mediante una regalía previamente pactada por la venta de la semilla de las variedades protegidas. En los convenios se establece que los productores de Tucumán no abonen las regalías por el uso de las variedades generadas por la EEAOC.

$\mathrm{Al}$ disponer únicamente del DOV para el recupero de la inversión, la inadecuada legislación de Argentina en la materia ha impedido el cobro de la mayor parte de las regalías que le hubiesen correspondido. A modo de ejemplo, se destaca que la variedad de soja más sembrada en el noroeste de Argentina es una desarrollada por este organismo, y mientras que de las siembras en Argentina prácticamente no se reciben pagos, la EEAOC ha recibido los correspondientes de parte de Paraguay, Bolivia y Brasil. Dado que la EEAOC no posee capacidad de gestión fuera de su provincia, en muchos casos las empresas asociadas son las encargadas del control de los derechos bajo diferentes sistemas de captura de valor, y esto es así en todos los casos fuera de Argentina, incluyendo los países limítrofes y Sudáfrica.

11 Síntesis elaborada a partir de la información proporcionada por el Dr. Daniel Ploper, director técnico de la EEAOC. 
Además, la EEAOC también tiene desarrollos en el campo de los bioinsumos, trabajando en marcos de convenios público/privados con una empresa, o público/público con el CONICET y la Universidad Nacional de Tucumán. Para estos casos, la gestión de los DPI se basa en patentes y una de ellas ya fue concedida en más de treinta países. Este bioinsumo fue luego licenciado y está siendo comercializado desde 2019 por una empresa para su uso en trigo, cebada y soja.

La EEAOC no hace uso de RRGG en sus proyectos de mejoramiento para variedades vegetales, pero sí indirectamente para el caso de bioinsumos. La gestión para su acceso y uso es llevada a cabo por el Centro Científico Tecnológico (CCT) del CONICET NOA Sur, quienes destacan que el proceso es difícil y burocrático, implicando acuerdos específicos que varían con cada provincia y que van acompañados de negociación de regalías de por medio. Este CCT es uno de los pocos organismos que ha podido finalizar algunos acuerdos, por lo que recibe consultas de otros institutos del CONICET, y ha propuesto a los ministerios de Ciencia y Tecnología y de Ambiente que se promuevan mecanismos armonizados, eficientes y expeditivos.

El primer punto en el análisis de estos cuatro casos deja en evidencia un fuerte argumento en contra de la hipótesis de Ridley (2010; 2020); no se observa que existan incentivos para realizar investigación que no pueda ser protegible y que eventualmente se transforme en innovación que esté en el dominio público. Además, exceptuando el caso de la PGM, que no se involucra, resulta claro al analizar los otros tres casos que los productos y procesos buscan orientarse hacia aquellos países que dispongan tanto del marco regulatorio más favorable como el de mejor alcance y cobertura de DPI. Desde una aproximación política, los casos parecen ser elocuentes ejemplos de que los DPI constituyen un incentivo y no un escollo para la innovación, lo cual ha sido reafirmado en otros estudios (Müller y Campos, 2020), y eso es lo que se ha constituido en el motor de la incentivación para la obtención (invención) y comercialización (innovación) de nuevas variedades vegetales (EuropaBio, 2018).

Además, y sin entrar a juzgar si la hipótesis de Ridley es comprobable en otras áreas, en el tema que nos ocupa -mejoramiento vegetal-sus argumentaciones son debatibles. Por ejemplo, sostener que la práctica de la domesticación empírica de especies que llevaron a cabo los seres humanos desde por lo menos 10000 ańos a. C. hasta Mendel haya sido la guía que lideró la elucidación matemática del mecanismo de la herencia genética por este notable botánico es una extrapolación con escaso asidero. Los experimentos de Mendel y sus acertadas conclusiones no constituyeron un proceso de invención acumulativa según las teorías de Karl Popper, sino un disruptivo cambio de paradigma más acorde con las teorías 
de Thomas Khun. En la misma línea, es posible señalar varios procesos inventivos disruptivos posmendelianos, desde las líneas puras en 1903 hasta la técnica de edición génica de CRISPR-Cas en 2012.

El segundo punto es que si bien los ejemplos son cuatro casos de innovación abierta en mejoramiento vegetal haciendo uso de los mecanismos de protección vigentes, la gestión de los DPI no sigue un patrón articulado uniforme. Así, en el modelo de la PGM, los DPI no forman parte de la vinculación. En el modelo de Bioheuris, los DPI se basan en la coexistencia, quedando a cargo de la primera aquello relacionado con patentes; y a cargo de la empresa semillera, lo relacionado con DOV. El modelo de Bioceres se sostiene en invenciones patentables, y el modelo de la EEAOC se basa en DOV para variedades gestionados generalmente por las empresas y en patentes para invenciones gestionados por la institución. En este aspecto se debe señalar un estudio realizado en España sobre 2800 pymes de todo tipo, que concluyó en que si bien estas no se benefician directamente de la innovación abierta o de las patentes de la misma forma que las empresas más grandes, sí lo hacen de formas alternativas de proteger la propiedad intelectual asociada a sus tecnologías patentadas (Brem, Nylund y Hitchen, 2017).

El tercer punto se relaciona con el modelo de coexistencia de derechos, el cual se sustenta en el supuesto de que las construcciones genéticas artificiales puedan ser protegibles mediante patentes. No necesariamente este criterio de patentabilidad se va a extender a las modificaciones de genes mediante edición génica ni mucho menos al producto final de mejoramiento asistido por marcadores moleculares, por lo cual, de no existir materia patentable, el modelo de protección mediante coexistencia deja de ser una alternativa. ${ }^{12}$ De darse este caso, las argumentaciones de Lizaso y Schötz (2017) en cuanto a la viabilidad de los nuevos tipos de estructuras organizacionales para procesos de innovación abierta en mejoramiento vegetal no podrían aplicarse.

El cuarto punto es que se demuestra que en mejoramiento vegetal los procesos

12 El 30 de enero de 2020, la compañía Arcadia Biosciences, Inc. anunció que la Oficina de Patentes de Australia le otorgó la patente número 2016288257 sobre un gen EPSPS editado para conferir tolerancia a glifosato en trigo. La compañía también recibió el aviso de concesión 15/740,876 de la Oficina de Patentes y Marcas de Estados Unidos. El trabajo realizado fue de elevado nivel técnico, ya que se editaron los tres genomas A, B y D del trigo codificando para una EPSPS que tiene una sustitución de treonina a isoleucina en la posición 168 de SEQ ID NO. 9, y, además, en el que una o más mutaciones no transgénicas inducidas por humanos contribuyen a que una planta de trigo tenga resistencia al glifosato en comparación con una planta de trigo de tipo salvaje. En síntesis, lograron trigo tolerante a glifosato no transgénico. Es bastante difícil imaginar que este tipo de concesiones de patentes se extienda masivamente en otros países. 
de innovación abierta pueden generarse en cualquier dirección, es decir, desde lo privado hacia lo público y viceversa, poniendo de manifiesto uno de los aspectos virtuosos de la herramienta.

El quinto punto es quizás el más relevante a mediano y largo plazo; en ninguno de los cuatro casos analizados se trató el tema del uso de RRGG dentro del concepto de innovación abierta para mejoramiento vegetal, lo cual tiene una lectura bipolar. Si los RRGG no forman parte del problema, es porque estos no están en el problema, cuando concretamente, en mejoramiento vegetal, deberían estar. Esto puede deberse a múltiples razones, comenzando por el hecho de que, a pesar de su importancia, las empresas no consideren necesario su acceso ni su uso. También es posible suponer que las empresas consideren que acceder y usar un RRGG es de tal complejidad administrativa que es preferible evitar su uso. ${ }^{13}$

Sea una cosa o la otra, lo que se observa es fuertemente indicativo de que los RRGG no están siendo utilizados o están siendo subutilizados, con la consiguiente estrechez de la base genética del mejoramiento y el acortamiento de las posibilidades de incremento de la tasa de productividad de los cultivos. Lo notable es que con las enormes posibilidades técnicas que brinda la conjunción del análisis genómico profundo, la información de la interacción genotipo/fenotipo y la edición génica se abren, como jamás había ocurrido con anterioridad, tanto las posibilidades corrientes de acceso y uso físico a los RRGG como el uso no físico de estos (información). En síntesis, parece estar ocurriendo una paradójica situación en la cual se han podido desarrollar una serie de metodologías de avanzada aplicables en mejoramiento vegetal moderno que podrían estar significativamente desaprovechadas (Henry, 2019).

Es necesario profundizar este último punto. Se debe reconocer que durante la etapa en la que el único modelo existente era la innovación cerrada, los DPI fueron considerados un oxímoron caracterizado por la protección total y el objetivo del bienestar general. La gravitación hacia modelos de innovación

13 Ver, por ejemplo, el caso descripto de la EEAOC para bioinsumos. Es posible agregar que, en dos oportunidades dentro del proyecto de la PGM, unidades académicas universitarias se acercaron a UBATEC S.A. para identificar potenciales interesados en proyectos de innovación vegetal a partir de recursos genéticos autóctonos. Contactadas varias importantes empresas alimenticias nacionales, sus respuestas para generar proyectos de innovación abierta fueron negativas. En ambos casos, esto se debió a malas experiencias anteriores de las empresas en proyectos de RRGG, derivadas tanto de las desmesuradas exigencias burocráticas de las regulaciones provinciales, en algunos casos, como en el desconocimiento de la regulación por parte de las autoridades, en otros, sumadas a las presiones de algunos grupos sociales descendientes de etnias nativas o grupos políticos de izquierda opuestos a cualquier tipo de proyecto de innovación con dichos recursos. 
abierta, colaborativos, compartidos, comunales e interdependientes ha sucedido en un mundo inmerso en el aumento exponencial de las tecnologías de la información y comunicación (TIC) y conquistado en parte por los movimientos de software libres. Cuando estas tendencias todavía no estaban claras, Oguamanam (2013) había correctamente predicho que el intercambio habitual de semillas como pieza central de la naturaleza abierta inherente al desarrollo ancestral de la agricultura, especialmente en las comunidades indígenas y locales, iba a quedar afectado por los marcos legales e institucionales emergentes para la gobernanza de los RRGG. La hipótesis es en extremo relevante; y su interpretación, a menudo, errónea; la utilización de los RRGG no está obstaculizada por los sistemas de DOV tipo UPOV (Unión Internacional para la Protección de las Obtenciones Vegetales, por sus siglas en francés) -ya que estos son tipo open source-, sino por los regímenes internacionales y legislaciones nacionales de RRGG que son todos, sin excepción, de tipo close source (Rapela, 2010; 2016).

En este sentido, probablemente la propuesta de Oguamanam (2013) relativa a que los DPI deben "calibrarse" para adaptarlos a los procesos de innovación abierta sería insuficiente. Parecería ser más correcto argumentar que todo el marco regulatorio es el que debe adaptarse para favorecer genuinos procesos de innovación abierta en mejoramiento vegetal moderno.

\section{Conclusiones}

Los procesos de innovación abierta aplicados al mejoramiento vegetal moderno disponen, comparativamente, de un alto grado de probabilidades de éxito en relación con los clásicos procesos de innovación cerrada. Resulta claro que en el marco de esta especialidad, en la que los requerimientos de conocimientos y la necesidad de generación de nuevas invenciones tanto en productos como en procesos es tan demandante y en las fronteras de la ciencia, no hay en el sector público ni en el privado capacidad tecnológica autárquica capaz de dominar por completo emprendimientos de este tipo. La colaboración publica/privada y privada/privada es la llave para fomentar la innovación.

A pesar de las oportunidades que genera el concepto de "innovación abierta aplicada al mejoramiento vegetal moderno", gravita una concreta externalidad negativa. Asumiendo que el mejoramiento vegetal moderno es de extensiva aplicación en instituciones públicas y en medianas y grandes empresas semilleras de países agrícolas industrializados y en vías de desarrollo, paradójicamente no se detecta un aumento en la tasa de innovación en mejoramiento vegetal medida por el incremento promedio de la productividad de los cultivos. No es aventu- 
rado pensar que una de las causas para explicar este relevante fenómeno podría radicar en que la base genética en la cual se está apoyando el mejoramiento vegetal moderno se ha estrechado de forma significativa. De ser así, aunque el mejoramiento vegetal moderno y los procesos de innovación abierta conformen una combinación virtuosa, esta no llegará a tener el éxito esperado si no incorpora un aprovechamiento masivo de la variabilidad genética existente.

En este escenario, los derechos de propiedad intelectual forman parte de las variables de la complicada ecuación y su interrelación con la técnica (mejoramiento vegetal moderno), con el proceso (innovación abierta) y con el acceso y uso de los recursos genéticos con el objetivo de fomentar la innovación está en discusión, abriéndose tres posibles hipótesis, no exhaustivas: 1) eliminar los derechos de propiedad intelectual aplicables sobre variedades vegetales e invenciones biotecnológicas en plantas; 2) gestionar de forma novedosa los derechos de propiedad intelectual haciendo uso del marco normativo vigente; o 3) desarrollar un nuevo sistema integrado sui generis de protección para todo tipo de germoplasma vegetal.

Para tratar de verificar estas tres hipótesis, se las contrastó con cuatro casos que si bien pueden parecer cuantitativamente escasos, son paradigmas concretos y reales en el tema analizado a nivel local.

En los casos se pudo apreciar una ausencia de evidencia directa o indirecta que permita probar la hipótesis 1 y suponer que los actores involucrados hayan considerado que la no aplicación de derechos de propiedad intelectual pueda ser una herramienta eficaz para los objetivos planteados.

También fue posible detectar que le elección de los caminos técnicos a recorrer, el tipo de especie vegetal para el trabajo, la separación de roles en la observancia del derecho y la elección de los países para los emprendimientos dependieron estrechamente de las herramientas de propiedad intelectual disponibles. En otras palabras, el marco legal no está siguiendo al avance tecnológico, sino que el avance tecnológico se orienta en la dirección en la que es posible protegerlo mediante algún mecanismo de propiedad intelectual. En tal escenario, la gestión novedosa de los derechos de propiedad (hipótesis 2) parecería factible, aunque tropieza con dos limitantes: 1) el alcance de los derechos (aún se está lejos de poder definir si los genes editados son materia patentable y si dos variedades vegetales de idéntico fenotipo, pero divergentes en genotipo, pueden ser consideradas diferentes o no); y 2) las excepciones al alcance de los derechos (si la nueva variedad editada protegida por un DOV es libre de ser usada por un tercero, ¿cómo se protege la edición puntual realizada?). Las limitantes citadas se traducen en que las últimas tecnologías genómicas aplicadas en mejoramiento vegetal 
están generando productos que muy probablemente no puedan ser protegidas de manera "eficaz" mediante ninguna de las herramientas de propiedad intelectual actualmente vigentes. ${ }^{14}$

Junto con ello, y aunque se esfuercen al límite las posibilidades de las hipótesis 1 y 2 , sigue insoluble el tema de la falta de uso o subutilización de los recursos genéticos y cómo incorporarlos masivamente al mejoramiento vegetal moderno en un proceso de innovación abierta. Esto no hace más que señalar la dificultad resultante de querer aplicar la enorme cantidad de tratados, convenios y acuerdos internacionales relacionados con la propiedad intelectual y el acceso/uso/beneficio compartido de los recursos genéticos a las variedades vegetales modernas cuando todos esos instrumentos del derecho internacional han sido redactados en una etapa pregenómica del mejoramiento vegetal.

La hipótesis 3 de desarrollar un sistema integral sui generis de protección para todo tipo de germoplasma vegetal, es decir, disponer de un nuevo sistema legal elaborado teniendo en cuenta los avances tecnológicos de la etapa genómica del mejoramiento vegetal, constituye una tercera alternativa. Si bien un sistema integral de este tipo ya ha sido propuesto, la funcionalidad de este para fomentar la innovación aún no ha sido probada.

Cuanto más tiempo se continúe en la situación actual, más difícil será encontrar la solución. Expresado en otros términos, mientras que la hipótesis 2 implica que la innovación debe orientarse hacia aquellas soluciones capaces de ser gestionadas en forma innovadora por algún sistema de propiedad intelectual vigente, aunque la protección esté limitada por la velocidad del avance tecnológico, la hipótesis 3 implica que el marco legal debe reformularse para acompañar la innovación de una manera integral. Una decisión crítica que en algún momento deberán tomar los responsables de las políticas tecnológicas de los países y que, sin dudas, impactará sobre la tasa de incremento productivo de los cultivos, o sea, la innovación.

14 La protección "eficaz" es lo que demanda taxativamente el artículo 27.3.b del ADPIC. Ley 24425/1994, "Aprobación del Acta final que incorpora la Ronda Uruguay de negociaciones comerciales multilaterales, declaraciones y entendimientos ministeriales y el Acuerdo de Marrakech, promulgada por decreto 2279/94", (BO 05/01/1995), Ministerio de Justicia y Derechos Humanos, Presidencia de la Nación. http://servicios.infoleg.gob.ar/infolegInternet/verNorma.do?id=799. 


\section{Bibliografía}

Aoki, K. (2009). "Free Seeds, Not Free Beer": Participatory Plant Breeding, OpenSource Seeds, and Acknowledging User Innovation in Agriculture. Fordham Law Review, 77(5), 22752310. https://ir.lawnet.fordham.edu/flr/vol77/iss5/9.

Baregheh, A., Rowley, J. y Sambrook, S. (2009). Towards a multidisciplinary definition of innovation. Management Decision, 47, 1323-1339.

Bledow, R., Frese, M., Anderson, N., Erez, M. y Farr, J. (2009). A dialectic perspective on innovation: Conflicting demands, multiple pathways, and ambidexterity. Industrial and Organizational Psychology, 2, 305-337.

Boer, H. y During, W. E. (2001). Innovation, what innovation? A comparison between product, process and organizational innovation. International Journal of Technology Management, 22, 83-109.

Book, J. (5 de mayo de 2020). Matt Ridley on Innovation. American Institute for Economic Research. https://www.aier.org/article/matt-ridley-on-innovation/.

Botana Agra, M. (2017a). Efectos de la Patente. En Fernández-Novoa, C., Otero Latres, J. M. y Botana Agra, M. (Eds), Manual de la Propiedad Industrial (3 $3^{\mathrm{a}}$ ed., pp. 163-178). Madrid/ Barcelona/Buenos Aires: Marcial Pons.

Botana Agra, M. (2017b). Protección jurídica de las obtenciones (variedades) vegetales. En Fernández-Novoa, C., Otero Latres, J. M. y Botana Agra, M. (Eds), Manual de la Propiedad Industrial (3 $3^{a}$ ed., pp. 319-344). Madrid/Barcelona/Buenos Aires: Marcial Pons.

Brem, A., Nylund, P. y Hitchen, E. (2017). Open innovation and intellectual property rights. Manag Decis, 55(6), 1285-1306.

Byrum, J. (febrero de 2017). Open Innovation Drives Progress in Plant Breeding. Seed World. https://seedworld.com/open-innovation-drives-progress-plant-breeding/.

Chesbrough, H. W. (2003). Open Innovation: The new imperative for creating and profiting from technology. Boston: Harvard Business School Press.

Chesbrough, H. W. (2006). Open Business Models: How to Thrive in the New Innovation Landscape. Boston: Harvard Business School Press.

Chesbrough, H. W. (enero de 2011). Open Services Innovation: Rethinking Your Business to Grow and Compete in a New Era. Nueva York: John Wiley \& Sons.

Chesbrough, H. W. (2019). Open Innovation Results: going beyond the hype and getting down to business. Londres: Oxford University Press.

Chesbrough, H. W. y Bogers, M. (2014). Explicating open innovation: clarifying an emerging paradigm for understanding innovation. En Chesbrough, H. W., Vanhaverbeke, W. y West, J. (Eds.), New frontiers in open innovation (pp. 1-37). Oxford: Oxford University Press.

Chesbrough, H. W, Vanhaverbeke, W y West, J. (Eds.) (2006). Open Innovation: Researching a New Paradigm. Londres: Oxford University Press.

Correa, C. M. (2014). Patent protection for plants: Legal options for developing countries (Research Paper 55). Ginebra: South Centre.

Correa, C. M. (2015). La protección de las obtenciones vegetales para los países en desarrollo. Una herramienta para el diseño de un sistema sui generis de protección de las obtenciones vegetales: una alternativa al Acta de 1991 del Convenio de la UPOV. APBREBES. http://www.apbrebes.org/.

Crossan, M. M. y Apaydin, M. (2010). A multi-dimensional framework of organizational innovation: A systematic review of the literature. Journal of Management Studies, 47, 1154-1191. 
La interacción entre los derechos de propiedad intelectual y los procesos de innovación abierta aplicados en el mejoramiento..

Dederer, H. G. (4 de junio de 2020). Patentability of Genome-Edited Plants: A Convoluted Debate. IIC - International Review of Intellectual Property and Competition Law, (51), 681-684. https://doi.org/10.1007/s40319-020-00952-9.

EuropaBio (2018). Biotechnology. How intellectual property rights promote innovation and create economic and societal value. https://www.europabio.org/sites/default/files/IP\%20brochure-\%20final.pdf.

Fernando Magarzo, M. R. y Lema Devesa, C. (2002). Las patentes sobre biotecnología en el Derecho español. Actas de derecho industrial y derecho de autor, (23), pp.147-168.

Hall, B. H. (2010). Open Innovation and Intellectual Property Rights. The Two-edged Sword. Japan Spotlight: Economy, Culture y History, (jan/feb), 18-19.

Henry, J. (2019). Innovations in plant genetics adapting agriculture to climate change. Current Opinion in Plant Biology, (13), 1-6.

Instituto Interamericano de Cooperación para la Agricultura. (2019). Innovación y Tecnología. Instituto Interamericano de Cooperación para la Agricultura. https://repositorio.iica.int/bitstream/handle/11324/7974/BVE19040251e.pdf; jsessionid=720035D979EFDB28CA06C$6112587 \mathrm{FF} 20$ ? sequence $=1$ yutm_source=email_marketingyutm_admin $=131431 \mathrm{yutm} \_\mathrm{me}-$ dium=emailyutm_campaign=hs_Arg_Encuentro_Virtual_EL_IMPACTO_CIENTFICO_ TECNOLGICO_EN_EL_DESARROLLO_DEL_SECTOR_AGROPECUARIO_OE.

Kalange, C. M. (2020). Role of Intellectual Property in Innovation and New Product Development, Consultor, División de Pymes de la OMPI. Sitio web de la Organización Mundial de la Propiedad Intelectual. https://www.wipo.int/export/sites/www/sme/en/documents/pdf/ ip_innovation_development.pdf.

Lema Devesa, C. y Fernando Magarzo, M.R. (2002). Las patentes sobre biotecnología en el derecho español. Actas de Derecho Industrial y de Derechos de Autor, XXIII, pp. 147-168.

Lizaso, F y Schötz, G. (Dirs.). (2017). Plataformas abiertas para tecnologías estratégicas: relevamiento, análisis de pre-factibilidad y propuesta de diseño de modelo conceptual: informe final ( $1^{\text {a }}$ ed.). Ciudad Autónoma de Buenos Aires: Ministerio de Ciencia, Tecnología e Innovación Productiva, Presidencia de la Nación. https:/www.argentina.gob.ar/sites/default/files/plataformas-abiertas-para-tecnologias-estrategicas_0.pdf.

Müller, M. L. y Campos, H. (2020). Open Innovation and Value Creation in Crop Genetics. En Campos, H. (Ed.), The Innovation Revolution in Agriculture. Springer, Cham. https://doi. org/10.1007/978-3-030-50991-0_3.

O’Sullivan, D. y Dooley, L. (2009). Applying innovation. Thousand Oakes: SAGE Publications.

Oguamanam, C. (2013). Open innovation in plant genetic resources for food and agriculture. Chicago-Kent Journal of Intellectual Property, 13(1), 11-50. https://papers.ssrn.com/sol3/papers.cfm?abstract_id=2310635.

Organization for Economic Co-operation and Development. (2005). Oslo Manual 2005: Guidelines for collecting and interpreting innovation data ( $3^{\mathrm{a}} \mathrm{ed}$.). París: Organization for Economic Co-operation and Development.

Organization for Economic Co-operation and Development. (2018). Oslo Manual 2018: Guidelines for Collecting, Reporting and Using Data on Innovation (4 $4^{\mathrm{a}}$ ed.). París: Organization for Economic Co-operation and Development. https://doi.org/10.1787/9789264304604-en.

Organization for Economic Co-operation and Development/Food and Agriculture Organization. (2019). OECD-FAO Agricultural Outlook 2019-2028. París: OECD Publishing/Roma: 
Food and Agriculture Organization of the United Nations. https://doi.org/10.1787/agr_outlook2019-en.

Pénin, J. y Neicu, D. (2018). Patents and open innovation: bad fences do not make good neighbors. Journal of Innovation Economics \& Management, (25), pp. 57-85.

Rapela, M. A. (2000). Derechos de propiedad intelectual en vegetales superiores. Buenos Aires: Editorial Ciudad Argentina.

Rapela, M. A. (2010). Farmer's exception, farmer's rights and other related issues. Seed News, $X I V(1)$, pp. 28-29.

Rapela, M. A. (2016). Ley 20.247 de Semillas y Creaciones Fitogenéticas: las razones para su actualización y los proyectos bajo análisis en Argentina. Revista Interdisciplinaria de Estudios Agrarios, (45), 69-98.

Rapela, M. A. (2019a). Post Malthusian dilemmas in Agriculture 4.0. En Rapela, M. A., Fostering innovation for Agriculture 4.0. A comprehensive Plant Germplasm System (pp. 1-16). Suiza: Springer Nature.

Rapela, M. A. (2019b). The regulatory tangle. En Rapela, M. A., Fostering innovation for Agriculture 4.0. A comprehensive Plant Germplasm System (pp. 17-52). Suiza: Springer Nature.

Rapela, M. A. (2020). Mejoramiento vegetal moderno, inteligencia artificial y derechos de propiedad intelectual. Revista Jurídica Austral, 1(2). En prensa.

Rapela, M. A. (Dir.) y Schötz, G. J. (Coord.). (2006). Innovación y propiedad intelectual en mejoramiento vegetal y biotecnología agrícola. Buenos Aires: Heliasta.

Ridley, M. (2010). The Rational Optimist. Estados Unidos: Harper.

Ridley, M. (2020). How Innovations Works and Why it flourishes in freedom. Estados Unidos: Harper.

Rogers, E. M. (2003). Diffusion of innovations (5 $\left.{ }^{\mathrm{a}} \mathrm{ed}.\right)$. Nueva York: Free Press.

Ruane, J. (Ed.). (2019). Proceedings of the International Symposium on Agricultural Innovation for Family Farmers. Unlocking the potential of agricultural innovation to achieve the Sustainable Development Goals. Roma: Food and Agriculture Organization of the United Nations. http:// www.fao.org/3/ca4781en/CA4781EN.pdf.

Sánchez Herrero, A. (2007). El contenido patrimonial del derecho de obtentor (Vol. 44). Córdoba: Ediciones de la Academia Nacional de Derecho y Ciencias Sociales de Córdoba.

Schilling, M. A. (2013). Strategic management of technological innovation (4a ed.). Nueva York: McGraw-Hill/Irwin.

Tidd, J. y Bessant, J. (2009). Managing innovation: Integrating technological, market and organizational change ( $\left.4^{\mathrm{a}} \mathrm{Ed}\right)$. Hoboken, Nueva Jersey: John Wiley \& Sons.

Williams, E. (2010). Intellectual Property Rights and Innovation: evidence from the human genome. Journal of Political Economy, 121(1), 1-27.

Virginia Tech. (16 de octubre de 2019). Accelerating global agricultural productivity growth is critical. ScienceDaily. www.sciencedaily.com/releases/2019/10/191016074750.htm. 
